\title{
Evidence of Sex-specific Differences in Masticatory Jaw Movement Patterns
}

\author{
G.E. Gerstner ${ }^{1,2}$ and V.V. Parekh ${ }^{2}$
}

${ }^{1}$ Department of Biologic and Materials Sciences, 3355 Dental Bldg., University of Michigan, Ann Arbor, Michigan 48109-1078; and ${ }^{2}$ Department of Psychology, University of Michigan, Ann Arbor, Michigan 48109-1109

\begin{abstract}
The complexity of human oral functional movements has not been studied in detail quantitatively, and only recently have studies begun to evaluate whether such movements contain sex-specific characteristics. Therefore, the purposes of this study were: (1) to quantify in detail the jaw movements and associated masticatory electromyographic activity occurring during gum chewing, and (2) to explore these data for evidence of sex specificity. Fourteen male and 17 female subjects participated in the study. Approximately 11 right- and 11 left-sided chewing cycles and associated masticatory electromyographic activity were sampled from each subject. The samples were quantified into 165 variables per chewing cycle, averaged to create a single multivariate vector for each subject, and then analyzed by a step-wise discriminant analysis. With a combination of 6 variables, a jackknifed cross-validation test found the probability of correct classification to be $93.5 \%$. These findings support the hypothesis that masticatory jaw movements contain sex-specific features.
\end{abstract}

Key words: mastication, sex, gender, human, discriminant analysis.

\section{Introduction}

Recently, there has been considerable interest in sex- and gender-specific differences in neurobehavioral phenomena. Neuroanatomists have found sex differences in the central nervous systems of laboratory animals (Gorski et al., 1978; Gorski, 1984; Hines et al., 1992) and humans (Gorski, 1978; Allen and Gorski, 1990, 1992; Allen et al., 1991; Aboitiz et al., 1992). These anatomical differences have been correlated with sex-specific behaviors in laboratory animals (Gorski, 1978; Hines et al., 1987, 1992; Raum et al., 1990), and investigators have argued that sex-specific brain differences account for gender-specific perceptual, cognitive, and motor differences (Hutt, 1978; Stoner, 1978; Allen and Gorski, 1990, 1992; Allen et al., 1991; Ames, 1991; Hines and Kaufman, 1994). Developmental psychologists have found compelling sex- and gender-specific differences in the behavior of infants, children, and adolescents (Goldberg and Lewis, 1969; Weisfeld, 1979; Zucker et al., 1982; Zuger, 1984; Phillips and Over, 1992; Hines and Kaufman, 1994; Alexander and Hines, 1994; Lobel, 1994), suggesting that sex specificity develops early. These neuroanatomical, neurobehavioral, and psychological studies represent important groundwork for our understanding of how genetic and proximate mechanisms interact with social and cultural parameters to mold individual male and female humans.

Unfortunately, little work has been published on whether oral motor behaviors contain sex-specific elements (cf. Korner, 1973; Sheikholeslam et al., 1980; Corruccini et al., 1985; Howell, 1987; Bakke et al., 1990). This is surprising, considering the clinical and basic scientific implications of such studies. For instance, significant sex differences exist in dentoskeletal morphological features of adult humans (Ingerslev and Solow, 1975; Tradowsky, 1990; Terk and Fenart, 1992), and oral function probably plays an important role in the development of these differences (Ingerslev and Solow, 1975; Long et al., 1982; Tradowsky, 1990; Terk and Fenart, 1992). In maturing rats, dentoskeletal morphology and occlusion can be significantly altered by sham lesions to the trigeminal motor nucleus and adjacent pontine reticular formation (Byrd et al., 1990), suggesting that altered function can lead to altered dentoskeletal morphology during development. If the developing dentoskeletal morphology and occlusion are influenced by jaw function, then sex- 
specific differences in oral function could influence dentoskeletal morphological development. Unfortunately, little is known about sex differences in human function; consequently, these important issues cannot be addressed.

Evidence shows that many types of orofacial pain and dysfunction have higher prevalence rates within a given sex. For instance, temporomandibular disorders (TMD) are more prevalent in females (Locker and Slade, 1988; Huber and Hall, 1990; Bakke and Møller, 1992; Krogstad et al., 1992; however, cf. Duckro et al., 1990; Glass et al., 1993). Furthermore, there is evidence that TMD symptoms are precipitated or perpetuated by functional and parafunctional habits (Christensen, 1971; Trenouth, 1979; Davidson and Mohl, 1987; Klineberg, 1988; Rugh and Harlan, 1988; Schiffman et al., 1992; Bakke and Møller, 1992; Krogstad et al., 1992; however, cf. Seligman and Pullinger, 1991). It is currently unknown whether women respond differently to pain precipitated by function and parafunction, or whether female function and parafunction are unique in ways that put women at higher risk. The only way for this issue to be evaluated is for oral function to be studied in detail for determination of whether sex-specific functional and parafunctional differences exist and whether these differences are linked to symptoms.

Despite the clinical and scientific implications of such work, little research has been done in this area. Several masticatory parameters appear to be sex-specific (e.g., Korner, 1973; Howell, 1987; Bakke, 1993); however, some of these parameters are sensitive to jaw size differences, and it is unclear how size effects were factored out of these previous studies' results. Nevertheless, previous findings are compelling and suggest that sex-specific masticatory features exist.

The purpose of the current study was to begin systematic investigations: (1) to develop a multivariate assay of oral function and parafunction, which assay is required to capture the richness and complexity of such phenomena; and (2) to determine whether sex-based differences in these phenomena exist. Because of our familiarity with masticatory jaw movements and associated electromyograms (EMG) (Gerstner and Goldberg, 1989, 1991, 1994; Gerstner et al., 1989; Gerstner, 1992), it seemed logical to begin such investigations by evaluating masticatory function first. We used a multivariate tool to analyze many variables simultaneously to find overt between-group differences and to enhance subtle between-group differences that would appear unremarkable in univariate analyses. Furthermore, we emphasized angular displacement measurements and used standardization methods to factor out jaw-sizedependency that may have confounded data sets in previous investigations. The current study also performed a crossvalidation test to gain inference regarding the generalizability of the findings.

\section{Materials and methods}

\section{Subjects}

Thirty-one healthy adults (14 males and 17 females) between the ages of 19 and 58 were studied. Descriptive statistics on these subjects appear in Table 1. Candidates were solicited from advertisements in local newspapers. Approximately 500 candidates responded by telephone to the advertisement and answered $\sim 30$ questions dealing with general health status, general dental health, orofacial pain experiences, etc. Candidates in good physical health and free from dental problems that required immediate treatment were asked to present to the laboratory for further evaluation and screening.

Candidates presenting to the laboratory underwent an extensive health history and clinical examination screening. The history and examination were a composite of the diagnostic tools used at the UCLA TMJ and Facial Pain Clinic, the University of Michigan Facial Pain Clinic, and recently published diagnostic criteria (Dworkin and LeResche, 1992). The health history evaluated general systems, head and neck sensorimotor systems, and dental, behavioral, and emotional health. It also included an anamnestic report of headaches, jaw, cheek, or temple pain, facial pain, and joint noises. Previous or current symptoms or signs were rated by candidates using visual analog scales (VAS).

Intra- and extra-oral examinations were performed on each candidate by a single clinician who had received training to standardize his examination skills (Goulet and Clark, 1990). The intra-oral examination evaluated the extant dentition, and overbite, overjet, and posterior cross-bite measures. The extraoral examination included standardized palpation of the masticatory musculature. TM joints were palpated while subjects chewed gum and while they performed a maximum jaw-opening task to document joint noises. Maximum opening, protrusive, and laterotrusive movements were measured with a ruler and evaluated for restrictions.

Results from the laboratory screening were used to develop the final subject pool. Candidates were excluded if: (1) their general health did not allow them to participate in the study; (2) they reported a history of orofacial pain (VAS scores $>25 / 100$ $\mathrm{mm}) ;(3)$ they lacked any centrals, laterals, canines, first molars, or second molars, and > 1 premolar per quadrant; (4) they reported a history of arthritis, TM joint noises, or other orofacial problems, (5) they had outstanding dental health problems; (6) they reported a history of organic disease; (7) they had used drugs that could interfere with affective or motor parameters (e.g., amphetamines, neuroleptics); (8) they had jaw movement restrictions (e.g., maximum opening $<40 \mathrm{~mm}$ ); or (9) they were $>15 \%$ overweight, thus making it difficult for clear EMG signals to be obtained.

Selected subjects included healthy individuals with no history of clinically evident signs or symptoms of orofacial pain or dysfunction. Subjects had to have $\geq 24$ teeth (missing third molars and orthodontically removed premolars were allowable conditions). All subjects had no anterior open bites or crossbites in centric occlusion. Subjects with a history of orthodontic treatment were included if such treatment had been completed $\geq 1 \mathrm{yr}$ prior to the study's onset. Clinical examination had to reveal no jaw movement restrictions and no TMJ noises. Of the 300 candidates who presented for the screening, 31 met these criteria and were used in the study.

Informed consents were obtained from subjects, and their rights and identities were protected. Only the general nature of the study was explained to the subjects-i.e., "we are studying gum chewing"-and to the clinicians and experimenters 
Table 1. Summary statistics of subjects by group

\begin{tabular}{|c|c|c|c|c|c|}
\hline Group & Count & Age $(\bar{x} \pm S D)^{a}$ & $\mathrm{Wt}(\overline{\mathrm{x}} \pm \mathrm{SD})^{\mathrm{b}}$ & Height $(\bar{x} \pm S D)^{c}$ & White:Non-white \\
\hline Female & 17 & $28.8 \pm 11.1$ & $133.3 \pm 23.9$ & $65.2 \pm 2.0$ & $7.5: 1$ \\
\hline Male & 14 & $28.0 \pm 10.7$ & $168.9 \pm 25.2$ & $70.9 \pm 1.9$ & $3.7: 1$ \\
\hline
\end{tabular}

a Mean ages of males and females were not significantly different (Mann-Whitney $U=104$, $z=-0.61, p=0.542$ ).

b Mean weights (lbs) of males and females were significantly different (Student's $t=-8.282$, $\mathrm{df}=29, \mathrm{p}<0.0001$ )

c Mean heights (in) of males and females were significantly different (Student's $t=-4.031, \mathrm{df}=29$, $\mathrm{p}<0.0004$ ).

d Ethnicities of the males and females were not significantly different $\left(x^{2}=0.531, \mathrm{df}=1, \mathrm{p}>0.4\right)$.

involved directly with the subjects. The protocols, above and below, were reviewed and approved by the appropriate institutional review board at the University of Michigan.

\section{Experimental set-up}

Each subject was seated comfortably on a chair facing a table. The table held experimental supplies and was used by subjects as an arm rest. Pairs of silver disc surface electrodes (Grass E6SH, Grass Instruments, Quincy, MA) were secured with electrode washers over the right and left anterior temporalis and superficial masseter muscles. A ground reference electrode (Grass E34D-S, Grass Instruments) was placed on the subject's left ear. EMG placement was determined by methods used in previous investigations (Rugh, personal communication). We reduced electrical impedance by cleansing the electrode placement areas with a light abrasive electrode preparation pad.

Two devices were used to track subjects' jaw and head movements. Each device consisted of three retroreflective markers mounted on a three-dimensional surface (Fig. 1, top). One device, attached to laboratory safety glasses, was used to track head movements. Glasses were checked for tightness of fit. If mobile, the glasses were secured to the subject's head with surgical tape. The device on the glasses was manipulated with orthodontic pliers so that the plane defined by the three retroreflective markers was approximately parallel to the subject's occlusal plane, i.e., the position of the posterior dentition's functional cusp tips. The use of this tracking device provided a means of subtracting head movements from the jaw movement data.

The second device was placed on the subject's lower left canine and was used to track jaw movements. The device consisted of three retroreflective markers attached to an orthodontic wire. This device was secured to the facial surface of the canine with a mini-twin orthodontic bracket and dental cement. The final experimental set-up is shown in Fig. 1.

\section{Experimental procedures}

Experimental procedures are shown in Fig. 2. The subject was given a gum base pellet (diameter, $\sim 8$ to $10 \mathrm{~mm}$; Wrigley's, Chicago, IL) and allowed to chew for several minutes. Two video cameras were then started and remained running continuously throughout the experiment. The subject was told to chew exclusively on the right side. After $\sim 10$ consecutive chewing cycles, an electronic signal was triggered, which started
EMG data collection and also recorded a square-wave pulse on the videotapes recording in both cameras. This trigger ensured that the onsets of digitized EMG and videotaped jaw movement data could be synchronized for analysis purposes. EMG data were digitized for $15 \mathrm{~s}$ after the trigger's onset, resulting in the acquisition of EMG data representing from 10 to 30 chewing cycles.

After completing these rightsided chewing cycles, the subject was told to place the gum on his/her tongue and clench in centric occlusion (Fig. 2). The clench provided a means of identifying the position of the jaw-tracking device with respect to the occlusal plane. This standard position was also used in the calculation of several variables. EMG data were not recorded during the clench.

After clenching for $\sim 10 \mathrm{~s}$, the subject was told to chew on the left side. After $\sim 10$ consecutive chewing cycles, an electronic trigger again started EMG data collection and recorded a square-wave pulse on the videotapes in both cameras. EMG data collection continued for $15 \mathrm{~s}$, after which time the experimental session ended. Thus, a session resulted in EMG and jaw movement data being acquired for 10 to 30 rightand 10 to 30 left-sided chewing cycles, as well as videotaped data of the subject during a maximum intercuspation clench.

\section{Jaw movement videotaping and digitization}

Head and jaw movements were videotaped with two genlocked video cameras (Panasonic 5100 HS camera, Panasonic AG 7400 SVHS recorder, Panasonic AG 455 camcorder, Peak Performance Event Synchronization Unit, Peak Performance Technologies, Inc., Englewood, $\mathrm{CO}$ ). The genlocking ensured that both cameras scanned video fields simultaneously so that no time-based errors occurred. A time code generator (SMPTE unit, Peak Performances Technologies, Inc.) was used to identify each video field on the video tapes. This ensured that no time-based errors occurred during digitization (see below). To avoid spatial errors in the data, we calibrated camera aspect ratios prior to the experiment (Peak 5.0 software, Peak Performance Technologies, Inc., Englewood, CO). This standardized the vertical and horizontal dimensional scales of each camera's video chip.

Cameras were positioned $\sim 45^{\circ}$ to the left and right of the subject's mid-sagittal plane (Fig. 1). Camera bodies were $\sim 0.3 \mathrm{~m}$ above the subject's Frankfort horizontal plane, and camera lenses were $\sim 2 \mathrm{~m}$ away from the subject's face. This configuration required that the cameras' optical axes be tilted slightly downward to tape the subject's midface and jaw. White balance, color balance, and focus were adjusted. Shutter speed was set to $1 / 1000 \mathrm{~s}$, and camera aperture was stopped down as far as possible to maximize depth of field without compromising image brightness. These steps ensured that videotaped jaw movement data remained clear and well-focused.

The positions of the 3 markers representing head movements and 3 markers representing jaw movements were 


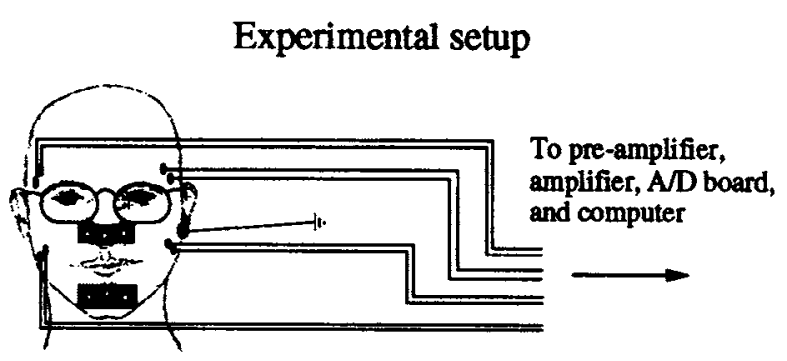

\section{Experimental arena}

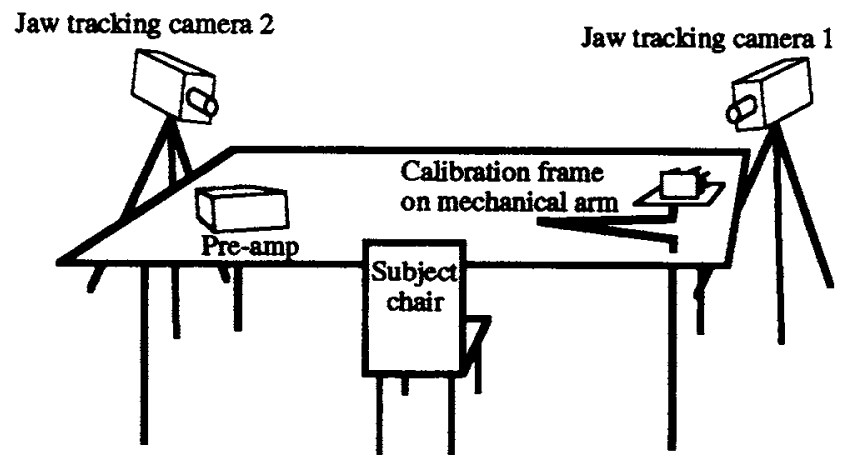

Figure 1. Experimental set-up and arena.

digitized (Peak Performance 3-D automatic motion analysis system, Peak Performance Technologies, Inc., Englewood, CO). Digitization began with the first videotape field in which the electronic trigger (see "Experimental procedures", above) occurred and continued for 900 consecutive fields, i.e., $15 \mathrm{~s}$ in real time. This 15-second interval corresponded with the time interval during which EMG data were digitized (Fig. 2).

\section{Jaw movement data processing}

Digitized head and jaw movement data were filtered by means of a fast Fourier transform algorithm (Peak 5.1.3 software, Peak Performance Technologies, Inc., Englewood, CO). This algorithm used the Jackson Knee Method to define optimal filtering parameters. This involved identifying and serially plotting the second derivative of the residuals for each filter parameter. Next, filter parameter triads were sampled consecutively until a triad was located whose sum $<0.1$. The filter parameter with the smallest value in this triad defined the cut-off point for filtering purposes.

Filtered data sets representing each camera view were converted to a three-dimensional data set by means of a direct linear transformation algorithm (Peak 5.0 software, Peak Performance Technologies, Inc., Englewood, CO). This algorithm required that a high-precision three-dimensional calibration frame be videotaped at the end of the recording session. To do this, the subject was moved out of the cameras' view-fields, and the calibration frame was placed in the same object space occupied by the subject during the experiment. The frame was then taped for $\sim 10 \mathrm{~s}$. After the subject was dismissed, 6 non-coplanar points on the calibration frame,

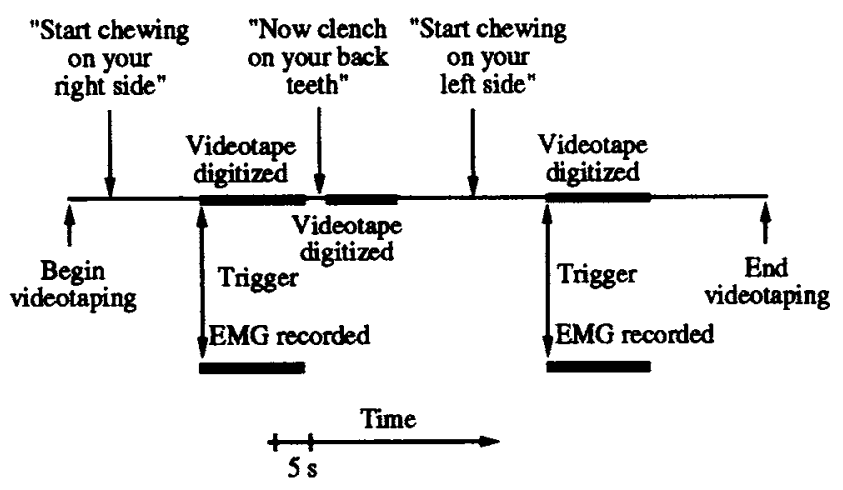

Figure 2. Experimental procedures. Shows verbal commands given to the subject during the experiment, when videotaping was performed, what videotape segments were digitized (thick lines), when the electronic signals were triggered, and when EMG data were digitized (see text).

whose coordinate values were known to $0.003 \mathrm{~mm}$, were digitized from images recorded on the videotapes. The direct linear transformation algorithm calculated the coordinate values of these 6 points as they appeared on the video chip surfaces of both cameras. The two camera views were compared, and three-dimensional coordinates for each point were calculated by a least-squares method to determine the best fit of the digitized values mapped onto the actual coordinate values of the points. Digitization of the calibration frame was redone if calibration accuracy was $>0.5 \%$ of the view-field. The direct linear transformation algorithm operated without investigators having to know the camera positions with respect to subjects' heads. Hence, it was not necessary for camera position between subjects to be standardized.

After the calibration frame points were digitized, the coordinate values of the digitized data were calculated. At this point, the data set defined the three-dimensional coordinate values of the markers representing head and jaw positions, and these coordinate values were accurate to at least $\pm 0.3 \mathrm{~mm}$.

An algorithm developed in the laboratory was used to subtract head movements from the jaw movement data (Fig. 3). This algorithm translated the data points so that the subject's right-most head marker became the origin, and then de-rotated the data around the $z-, y-$, and $x$-axes, respectively. As a result, the 3 head markers came to lie in the $x-z$ plane, and the 2 lateralmost head markers came to lie on the x-axis. This procedure was equivalent to having the subject's head immobilized, with the occlusal plane parallel to the $x-z$ plane and the subject's mid-sagittal plane parallel to the $y-z$ plane.

\section{EMG data acquisition, digitization, and processing}

EMG data were electronically amplified (total gain $=10,000)$ and high-pass-filtered $(20 \mathrm{~Hz})$. Preliminary power spectrum analyses revealed no significant frequency components $>600 \mathrm{~Hz}$; therefore, EMG data were digitized at $1200 \mathrm{~Hz}$ (Peak Performance A/D Interface Unit and software, Peak Performance, Inc., Englewood, CO; IBM-compatible 386/87 computer). Since $>90 \%$ of the energy content of the digitized EMG power density spectra were $<200 \mathrm{~Hz}$, data were then 

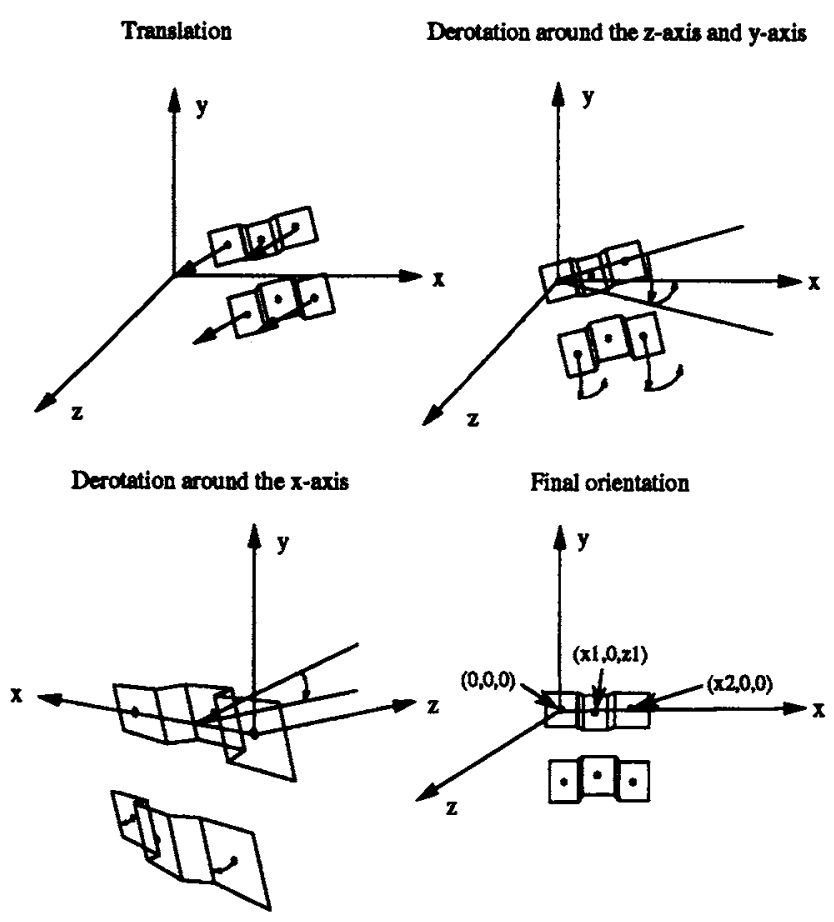

Figure 3. Schematic of the process used to translate and de-rotate the coordinate points of the retroreflective markers to remove head movements from the jaw movement data

further band-pass-filtered (from 20 to $200 \mathrm{~Hz}$ ), as well as notchfiltered $(60 \mathrm{~Hz})$, and full-wave-rectified (Datapac II software, RUN Technologies, Laguna Hills, CA). Electrical signals of known voltages (from $50 \mu \mathrm{V}$ to $1 \mathrm{mV}$ ) were digitized in the same manner as the EMG signals and served to calibrate EMG data.

\section{Data quantification and analysis}

EMG and jaw movement data were quantified chewing cycle by chewing cycle. For purposes of this study, a chewing cycle began at the previous cycle's point of maximum jaw closure and ended at the subsequent point of maximum jaw closure. Maximum jaw closures were determined with an algorithm that searched for the maximum vertical ( $y$-axis) value of the three-dimensional jaw movement data set (see "Jaw movement data processing", above) between two successive chewing cycles. Waveform segments occurring between two successive chewing cycles were visually identified for the algorithm by trained investigators.

Once a cycle's onset and offset had been determined, another algorithm was used to search for maximum jaw opening, i.e., the minimum y-axis value occurring during a cycle. Other algorithms determined the maximum left-lateral, right-lateral, anterior, and posterior jaw excursions by finding the respective maximum and minimum point values in the horizontal and anteroposterior axes. The times at which minimum and maximum velocity and acceleration occurred(1) during each cycle, (2) during the jaw-opening portion of a cycle, and (3) during the jaw-closing portion of a cycle-were similarly determined by an algorithm created in the laboratory to search for these extrema in the waveforms created by differentiation of the original jaw movement data set.

EMG burst onsets and offsets were identified by means of a sophisticated set of algorithms (Acceptance criteria selection module, Datapac II software, RUN Technologies, Laguna Hills, CA). Briefly, these algorithms identified candidate burst onsets and offsets based on whether the rectified EMG waveform crossed a user-defined threshold in a positive or negative direction, respectively. For candidate bursts to be accepted as actual bursts, other algorithms were used to monitor voltage ranges and duration ranges of the waveform. Waveforms that did not meet the acceptance criteria were excluded from consideration as bursts. Burst onsets and offsets that were not excluded by the acceptance criteria algorithms were evaluated visually against a plot of the EMG waveform, and artifacts that had been incorrectly identified as onsets and offsets were removed from analysis.

For identification of EMG peak amplitude, an algorithm created in the laboratory searched each EMG data set for the maximum voltage value of the waveform segment between a given burst's onset and offset times. The time at which the maximum voltage value occurred was used to define that burst's peak amplitude latency. Peak amplitude latencies were determined for each burst occurring in each of the 4 EMG data sets.

EMG and jaw movement data were quantified in terms of 165 variables per chewing cycle. A computer program created in the laboratory calculated the 165 variable measurements for each cycle for each subject. This quantification resulted in a $165 \mathrm{x} \mathrm{n}$ matrix, where $n=$ number of chewing cycles sampled during the two 15 -second trials. We reduced this matrix to a $1 \times 165$ vector by calculating the mean of each variable for the $n$ chewing cycles sampled for a given subject. Since one vector was created for each subject, 31 vectors were statistically analyzed.

The 31 vectors were grouped according to the respective subject's sex and analyzed by a step-wise discriminant analysis (BMDP Dynamic-7M software, BMDP Statistical Software, Inc., Los Angeles, CA) with use of an F-to-enter $\geq 4.0$ and an F-toremove $\leq 3.996$. A tolerance limit of 0.25 prevented variables having high squared multiple correlation with already-entered variables from being entered into the discriminant function. The maximum number of steps was specified as 6 to ensure that sample size $(n=31)$ was $>5$ times the number of variables entered into the discriminant function. A jackknifed crossvalidation test was performed to reduce the bias in the calculated posterior probabilities.

\section{Results}

Fig. 4 shows averaged jaw movement and EMG data (Signal averaging analysis module, Datapac II software, RUN Technologies, Inc.) from one subject and identifies 7 jaw movement landmarks and 12 EMG landmarks used to create variables. An additional 32 landmarks (not shown) were also used to create variables. These included the points at which maximum and minimum jaw movement velocity and acceleration occurred during (a) jaw opening and (b) jaw closing as described in the subject's (1) horizontal axis, (2) vertical axis, (3) anteroposterior axis, and (4) resultant vector space (32 landmarks $=2$ extrema $\times 2$ kinematic measures $\times 2$ cycle phases $x 4$ spatial parameters).

Rectangular boxes (Fig. 4) show examples of 
measurements used to create certain variables (Table 2). "Max. vert. dim." = the linear vertical measurement in $\mathrm{mm}$ from maximum closed position (calculated by averaging the $y$ axis values of $\mathrm{mc} 1$ and $\mathrm{mc} 2$ ) to maximum open ( $y$-axis value of mo) of a chewing cycle (see rows 24 and 25, Table 2). "mlc onset latency" (Fig. 4) indicates the point in time at which mlc occurred, where $t(0)=$ mcl (see row 2, Table 2). "Burst durations" (Fig. 4) were determined for each muscle by calculating EMG off latency, where $t(0)=E M G$ on (see row 6 , Table 2 ).

Fig. 5 shows examples of angular measurements used to create other variables (rows 26 to 29, Table 2). Diagrammatic representations of frontal $(A)$ and sagittal plane (B) views of a subject are shown with the jaw-tracking device in centric occlusion and in a position representing one of 7 landmarks described in Fig. 4. Insets show the position of the landmarks on a diagrammatic chewing cycle in the frontal (A) and sagittal (B) planes.

Table 2 lists the 165 variables, including temporally defined (rows 1 to 23 ) and spatially defined (rows 24 to 29 )
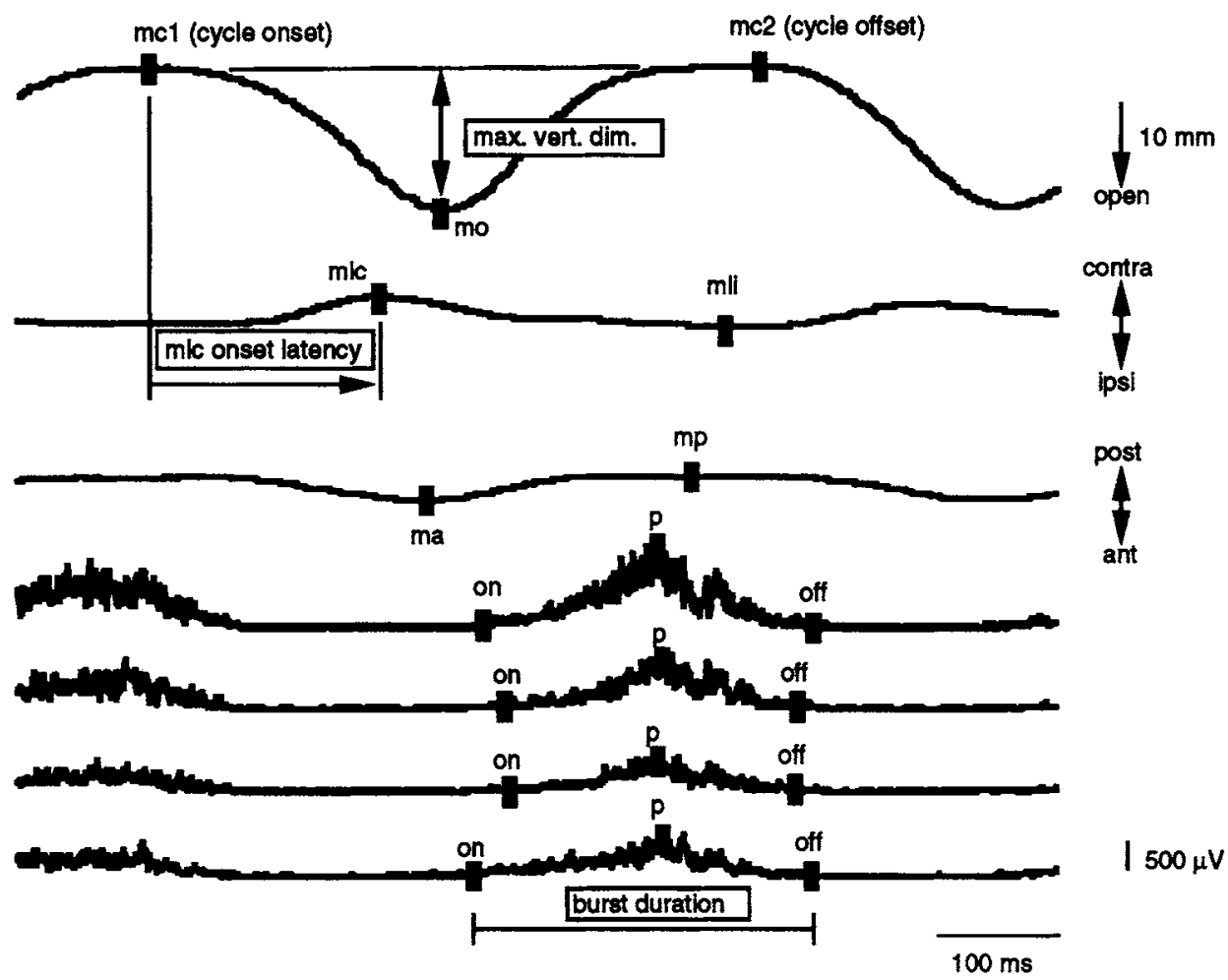

Figure 4. Plot of averaged jaw movement cycles and associated EMG activity. Top to bottom: vertical, horizontal, \& anteroposterior jaw movement components, ipsilateral \& contralateral temporalis muscle EMG activity, and ipsilateral \& contralateral masseter muscle EMG activity. Ipsilateral $=$ chewing side. Jaw movement plots are drawn to the same vertical scale as are the 4 EMG plots (see scales, right). Examples of ways in which variables were defined are indicated by rectangular boxes (see text). Landmark key: $\mathrm{mc1}=$ maximum jaw closure point at cycle onset; mo = maximum jaw opening point; $\mathrm{mc} 2=$ maximum jaw closure point at cycle offset; mlc $=$ maximum lateral jaw excursion point to the balancing side; $\mathrm{mli}=$ maximum lateral jaw excursion point to the working side; $\mathrm{ma}=$ maximum anterior jaw excursion point; $\mathrm{mp}=$ maximum posterior jaw excursion point; on = EMG burst onset; $\mathrm{p}=\mathrm{EMG}$ peak amplitude; and off = EMG burst offset.

variables. Note that spatially defined variables were standardized (rows 24, 25) or described as angular measurements (rows 26 to 29) in an attempt to control for size differences that existed between males and females (Table 1). All 165 variables were definable for each complete chewing cycle sampled; hence, no data were discarded as a consequence of the detailed, multivariate methods used in the study.

Significant sex-specific chewing differences emerged in the discriminant analysis. Table 3 summarizes the step-wise discriminant analysis results and includes results from test statistics performed on the data. The "U-statistic" (column 4) or Wilks' lambda is a multivariate analysis of variance that tests the equality of group means for the variables included in the discriminant function at each step (Dixon, 1992). This multivariate index varies between 0 and 1 and provides a statistical basis for determining if the degree of separation between the two subject groups is larger than what would be expected by chance. The U-statistic was transformed to the less arcane F-statistic (column 5) to simplify data interpretation. Male and female chewing patterns were significantly different based on the linear combination of the 6 variable measurements $(\mathrm{p}<0.0005$, step 6, Table 3).
The discriminant analysis generated two functions, a male and a female function, that were weighted sums of the 6 variables included in each function. Table 4 lists each variable's weighting coefficient and the constant used by each function. To validate a discriminant analysis, one enters the variable measures representing a subject into both discriminant functions. Subjects are assigned to the group with the highest posterior probability, e.g., if the male function generates a larger sum than the female function for a given subject's data, the data are classified as male. New data from future investigations can be plugged into the equations to test the generalizability of these results.

Toward this end, Table 5 shows jackknifed crossvalidation test results (Dixon, 1992). Column 1 designates the known group, and columns 3 and 4 tabulate the number of subjects classified into the groups by the analysis. The probability of correct classification for the discriminant analysis was $93.5 \%$. Note that only one male and one female were misclassified. The inference is that future generalized validation tests will continue to provide $\sim 93 \%$ separation between male and female data.

Since the male and female subjects differed significantly in terms of height and weight (Table 1), it was important to determine whether subject size contributed to the between-sex 
Table 2. Variable descriptions

\begin{tabular}{|c|c|c|}
\hline Row & Variable Descriptions or Derivations ${ }^{\mathrm{a}}$ & \# Variables \\
\hline 1 & Chewing cycle duration (i.e., latency to $\mathrm{mc} 2 ; \mathrm{t}[0]=\mathrm{mc} 1$ ) & 1 \\
\hline 2 & Latency to $\mathrm{ma}, \mathrm{mo}, \mathrm{mli}, \mathrm{mlc}, \mathrm{mp} ; \mathrm{t}(0)=\mathrm{mcl}$ & 5 \\
\hline 3 & (Latency to $\mathrm{ma}, \mathrm{mo}, \mathrm{mli}, \mathrm{mlc}, \mathrm{mp} ; \mathrm{t}[0]=\mathrm{mc} 1) \div$ chewing cycle duration & 5 \\
\hline 4 & EMG on latency; $t(0)=m c 1, m o, m c 2$ & $\begin{array}{c}12 \\
(4 \text { muscles } \times 3+[0])\end{array}$ \\
\hline 5 & EMG p latency; $t(0)=m c 1, m o, m c 2, E M G$ on & $\begin{array}{c}16 \\
(4 \text { muscles } \times 4 t[0])\end{array}$ \\
\hline 6 & EMG off latency; $t(0)=\mathrm{mc} 1, \mathrm{mo}, \mathrm{mc} 2, \mathrm{EMG}$ on & 16 (see above) \\
\hline 7 & EMG $p$ latency $\div E M G$ off latency; $t(0)=E M G$ on & 4 \\
\hline 8 & Latency to max., min. jaw velocity (3-dimensional); $\mathrm{t}(0)=\mathrm{mc} 1$ & 2 \\
\hline 9 & $\begin{array}{l}\text { Latency to max., } \mathrm{min} \text {. jaw velocity during jaw opening (horizontal, vertical, } \\
\text { anteroposterior axes separately); } \mathrm{t}(0)=\mathrm{mc} 1\end{array}$ & $\begin{array}{c}6 \\
(2 \text { extrema } \times 3 \text { axes })\end{array}$ \\
\hline 10 & $\begin{array}{l}\text { Latency to max., min. jaw velocity during jaw closing (horizontal, vertical, } \\
\text { anteroposterior axes separately); } \mathrm{t}(0)=\text { mo }\end{array}$ & 6 (see above) \\
\hline 11 & (Latency to max., min. jaw velocity [3-dimensional]; $\mathrm{t}[0]=\mathrm{mcl}$ ) $\div$ chewing cycle duration & 2 \\
\hline 12 & $\begin{array}{l}\text { (Latency to max., min. jaw velocity during jaw opening [horizontal, vertical, } \\
\text { anteroposterior axes separately]) } \div \text { latency to } \mathrm{mo} ; \mathrm{t}(0)=\mathrm{mcl}\end{array}$ & $\begin{array}{c}6 \\
(2 \text { extrema } \times 3 \text { axes })\end{array}$ \\
\hline 13 & $\begin{array}{l}\text { (Latency to max., min. jaw velocity during jaw closing [horizontal, vertical, } \\
\text { anteroposterior axes separately] } \div \text { latency to } \mathrm{mc} 2 ; \mathrm{t}(0)=\text { mo }\end{array}$ & 6 (see above) \\
\hline 14 & Latency to max., min. jaw acceleration (3-dimensional) during jaw opening, jaw closing; $\mathrm{t}(0)=\mathrm{mc1}$ & $\begin{array}{c}4 \\
\text { (2 extrema x } 2 \text { cycle phases) }\end{array}$ \\
\hline 15 & $\begin{array}{l}\text { Latency to max., min. jaw movement acceleration during jaw opening (horizontal, vertical, } \\
\text { anteroposterior axes separately); } t(0)=\mathrm{mc} 1\end{array}$ & $\begin{array}{c}6 \\
(2 \text { extrema } \times 3 \text { axes })\end{array}$ \\
\hline 16 & $\begin{array}{l}\text { Latency to max., min. jaw movement acceleration during jaw closing (horizontal, vertical, } \\
\text { anteroposterior axes separately); } t(0)=\text { mo }\end{array}$ & 6 (see above) \\
\hline 17 & $\begin{array}{l}\text { (Latency to max., min. jaw acceleration [3-dimensional] during jaw opening, jaw closing; } \\
\mathrm{t}[0]=\mathrm{mc} 1) \div \text { chewing cycle duration }\end{array}$ & $\begin{array}{c}4 \\
(2 \text { extrema x } 2 \text { cycle phases) }\end{array}$ \\
\hline 18 & $\begin{array}{l}\text { (Latency to max., min. jaw movement acceleration during jaw opening [horizontal, vertical, } \\
\text { anteroposterior axes separately]) } \div \text { latency to mo; } t(0)=\mathrm{mc} 1\end{array}$ & $\begin{array}{c}6 \\
(2 \text { extrema } \times 3 \text { axes })\end{array}$ \\
\hline 19 & $\begin{array}{l}\text { (Latency to max., min. jaw movement acceleration during jaw closing [horizontal, vertical, } \\
\text { anteroposterior axes separately] } \div \text { latency to } \mathrm{mc} 2 ; \mathrm{t}(0)=\mathrm{mo}\end{array}$ & 6 (see above) \\
\hline 20 & Difference in EMG on times between anatomically paired, side-paired muscles & 4 \\
\hline 21 & Difference in EMG p times between anatomically paired, side-paired muscles & 4 \\
\hline 22 & Difference in EMG off times between anatomically paired, side-paired muscles & 4 \\
\hline 23 & Difference in EMG burst durations between anatomically paired, side-paired muscles & 4 \\
\hline 24 & Max. vertical dimension of cycle $\div$ max. lateral dimension of cycle & 1 \\
\hline 25 & Max. vertical dimension of cycle $\div$ max. anteroposterior dimension of cycle & 1 \\
\hline 26 & Angle $\theta_{1}$, where leg 2 intersects jaw marker@ mc1, mli, mo, mlc, mc2, ma, mp & 7 \\
\hline 27 & Angle $\theta_{\mathrm{g}^{\prime}}$ where leg 2 intersects jaw marker @ $\mathrm{mc} 1, \mathrm{mli}, \mathrm{mo}, \mathrm{mlc}, \mathrm{mc} 2, \mathrm{ma}, \mathrm{mp}$ & 7 \\
\hline 28 & Angle $\theta_{\mathrm{a}-\mathrm{p}}$, where leg 2 intersects jaw marker $@ \mathrm{mc} 1, \mathrm{mli}, \mathrm{mo}, \mathrm{mlc}, \mathrm{mc} 2, \mathrm{ma}, \mathrm{mp}$ & 7 \\
\hline 29 & Angle $\theta_{\mathrm{t}}$, where leg 2 intersects jaw marker @ mc1, mli, mo, mlc, mc2, ma, mp & 7 \\
\hline
\end{tabular}

a Key: anatomically paired muscles = working- and balancing-side temporalis muscles, or working- and balancing-side masseter muscles; side-paired muscles = temporalis and masseter muscles both on the working side, or temporalis and masseter muscles both on the balancing side; $\max .=$ maximum; $\min .=$ minimum. See text and Figs. 4,5 for descriptions and references to mo, ma, $\mathrm{mp}, \mathrm{mli}, \mathrm{mlc}, \mathrm{mc} 2$, mc1, on, $\mathrm{p}$, off, and angles $\theta_{1}, \theta_{\mathrm{g}^{\prime}} \theta_{\mathrm{a}-\mathrm{p}^{\prime}}$ and $\theta_{\mathrm{t}}$.

differences. To evaluate the effects due to subject size, we calculated Pearson's coefficients of correlation using subject height as an index of size (Table 6). "Dependent" variables used in this correlation were those included in the discriminant analysis that also showed indications of significant betweengroup differences based on F statistics computed from a oneway analysis of variance for each individual variable. Two variables met these criteria; however, neither of these showed significant correlations with subject height (Table 6).

\section{Discussion}

The current study hypothesized that mastication would show characteristic differences between male and female subjects. The study used a step-wise multivariate procedure and showed that 6 variables contributed to a quantitative distinction between female and male masticatory patterns. The probability of correct classification was $93.5 \%$, with only 1/17 females and 1/14 males being misclassified (Table 5). 
The difference between male and female chewing patterns, based on the linear combination of 6 variable measures (Tables 3,4$)$, was highly significant $(p<0.0005)$. These significant differences appeared to be independent of size differences that existed between the males and females (Table 6). These results support the hypothesis that sexspecific characteristics exist in masticatory jaw movements.

How are such complex, multidimensional differences to be interpreted? Recent neurophysiologic work suggests that neural activity patterns do not correspond to simple "parameterized" models of movement (for review, see Fetz, 1992). In other words, it is arguable that neural activity patterns do not map in any simple way onto univariate descriptions of movement data. Thus, if sex-specific differences exist within the central neural systems involved in oral movement production, the manifestation of these differences in oral movements is likely to be complex. This is one reason why the current study used a multivariate statistical tool to search for sex differences. Future work will need to evaluate whether multidimensional movement parameters correlate with neural activity patterns, and whether such parameters provide more comprehensive insight into neural activity patterns than do univariate movement parameters. Standard neurophysiological methods could be used to this end, with the exception that multidimensional movement parameters would be used as the dependent variable.

On the other hand, previous investigations have reported sex differences in univariate measures of masticatory patterns (Neill and Howell, 1986; Howell, 1987). These studies found that vertical gape, maximum anterior jaw excursion, and maximum lateral jaw excursions during chewing were greater in men, and that women chewed more slowly than men. The current study also measured vertical gape (Angle $\theta_{\mathrm{g}}$ @ mo, row 27, Table 2), maximum anterior jaw excursion (Angle $\theta_{\text {a-p }} @$ ma, row 28, Table 2), maximum lateral jaw excursion (Angle $\theta_{1} @$ mli and mlc, row 26, Table 2), and chewing rate (row 1, Table 2). Vertical gape, maximum anterior jaw excursion, and maximum lateral jaw excursion were also quantified as ratios of each other (rows 24, 25, Table 2). However, none of these variables was included in the discriminant function. Furthermore, at step 0 of the discriminant analysis (not shown), the F statistic for each variable was computed from a one-way analysis of variance, and in no case did any of these variables show significant sexrelated differences ( $p>0.5$ ). An explanation for why the gape
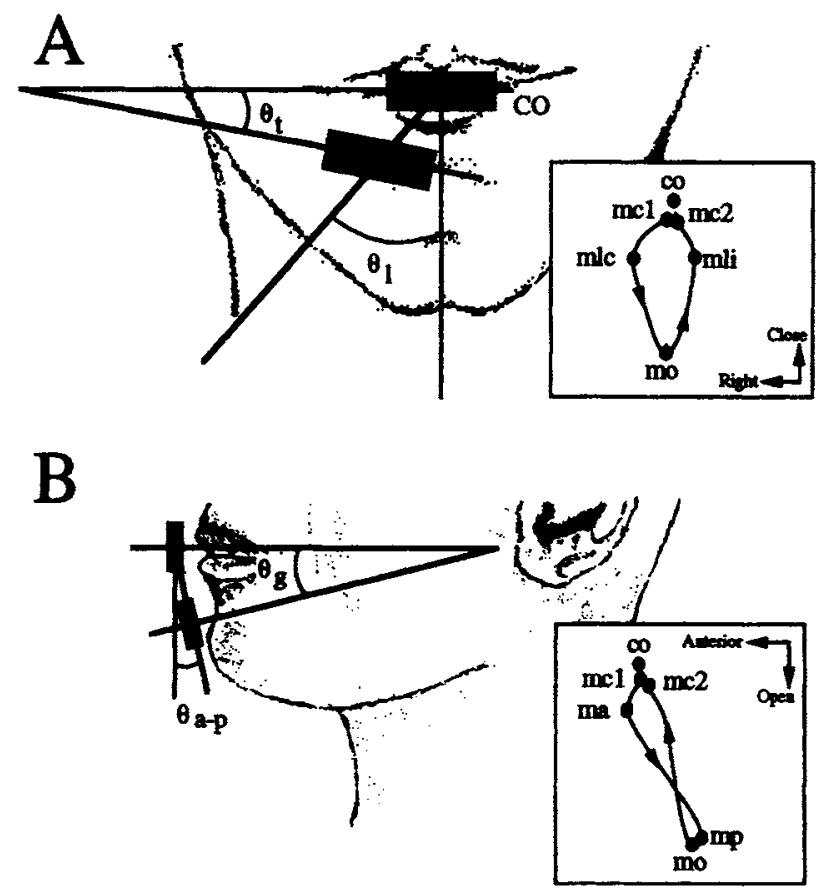

Figure 5. Diagram of the 4 angle types used in defining some variables (Table 2). Insets show representations of a chewing cycle in the frontal (A) and sagittal (B) planes. Abbreviations are the same as those used in Fig. 4, with one addition: $\mathrm{CO}=$ centric occlusion. (A) Angle $\theta_{t}$ : frontal plane angles. Legs of $\theta_{t}$ defined by the position of: (1) the two lateral-most markers when jaw was in $\mathrm{CO}$, and (2) the same markers when jaw was at each of the maximum jaw excursion points. Angle $\theta_{1}$ : frontal plane angles. Legs of $\theta_{1}$ defined by: (1) a line perpendicular to the occlusal plane and intersecting the center marker when jaw was in $\mathrm{CO}$, and (2) a line intersecting the center marker when jaw was in $\mathrm{CO}$, and the same marker when jaw was at each of the maximum excursion points. (B) Angle $\theta_{\mathrm{g}}$ : sagittal plane angles. Legs of $\theta_{g}$ defined by: (1) the position of the central and right lateral markers when the jaw was in $\mathrm{CO}$, and (2) the position of the same markers when the jaw was at each of the maximum jaw excursion points. Angle $\theta_{\mathrm{a}-\mathrm{p}}$ : sagittal plane angles. Legs of $\theta_{\mathrm{a}-\mathrm{p}}$ defined by: (1) a line perpendicular to the occlusal plane and intersecting the center marker when jaw was in $\mathrm{CO}$, and (2) line intersecting the center marker when jaw was in $\mathrm{CO}$, and the same marker when jaw was at each of the maximum excursion points.

and excursion variables were not significantly different is that angle and ratio measures were used in the current study, whereas linear displacement measures were used in previous studies. Linear displacement measures are sensitive to jawsize parameters, whereas angle and ratio measures are less

Table 3. Discriminant analysis summary results

\begin{tabular}{|c|c|c|c|c|c|c|}
\hline \multirow{2}{*}{ Step } & \multirow{2}{*}{$\begin{array}{l}\text { Variable Entered/Removed }{ }^{a} \\
\text { Angle } \theta_{\text {a-p }} @ \mathrm{mc} 2 \text { (row 28) }\end{array}$} & \multirow{2}{*}{$\begin{array}{c}\begin{array}{c}\text { F-to Enter/ } \\
\text { Remove }\end{array} \\
12.16\end{array}$} & \multirow{2}{*}{$\begin{array}{c}\begin{array}{c}\mathrm{U}- \\
\text { statistic }\end{array} \\
0.705\end{array}$} & \multirow{2}{*}{$\begin{array}{c}\text { Approx. } \\
\text { F-statistic } \\
12.156\end{array}$} & \multicolumn{2}{|c|}{$\begin{array}{c}\text { Degrees of } \\
\text { Freedom }\end{array}$} \\
\hline & & & & & 1 & 29 \\
\hline 3 & Latency to $\mathrm{mli} \div$ cycle duration (row 3 ) & 6.50 & 0.482 & 9.656 & 3 & 27 \\
\hline 4 & Latency to max. vertical acceleration during opening (row 15) & 7.89 & 0.370 & 11.073 & 4 & 26 \\
\hline 5 & Difference in ipsi. temp. \& mass. EMG off times (row 22) & 4.30 & 0.316 & 10.831 & 5 & 25 \\
\hline
\end{tabular}

Rows correspond to variable locations in Table 2. See also Figs. 4, 5 for further variable descriptions. 
Table 4. Variables included in the discriminant functions and their coefficients

\begin{tabular}{|c|c|c|}
\hline Variable Name & Female Function & Male Function \\
\hline Angle $\theta_{\mathrm{a}-\mathrm{p}} @ \mathrm{mc} 2$ (row 28) & 1.43885 & 7.79842 \\
\hline Latency to max. acceleration during closing $\div$ cycle duration (row 17 ) & 117.91041 & 179.79466 \\
\hline Latency to $\mathrm{mli} \div$ cycle duration (row 3 ) & 64.79659 & -10.51226 \\
\hline Latency to max. vertical acceleration during opening (row 15) & 0.05381 & 0.08566 \\
\hline Difference in ipsi. temp. \& mass. EMG off times (row 22) & 0.19625 & 0.05935 \\
\hline Latency to min. a-p. velocity during opening (row 9) & 0.02236 & 0.05471 \\
\hline Constant & -58.40467 & -67.16587 \\
\hline
\end{tabular}

a

Rows correspond to variable locations in Table 2.

sensitive. It is unclear how jaw-size effects were removed in previous studies; hence, previously reported differences may have reflected jaw-size effects as opposed to sex-specific effects. Whatever the case, it is clear that the parameters previously reported as being sex-specific may not be universally sex-specific.

Another explanation for the lack of sex specificity in univariate measures may have to do with the use of gum. Previous studies reported that gum reduces the level of sex specificity in mastication (Neill and Howell, 1986; Howell, 1987). It will be important to repeat the current study's methods on a new subject sample and to use foodstuffs of different consistencies. This will serve two purposes: (1) to determine whether the current study's discriminant functions (Table 4) will consistently distinguish males from females regardless of foodstuffs, and (2) to determine whether previously reported, univariate sex-specific distinctions (Neill and Howell, 1986; Howell, 1987) will emerge as a result of the use of non-gum food items.

Sex-specific differences reported in the current and previous studies may be due to genetic, neurophysiological, dentoskeletal morphological, or sociocultural parameters. However, correlations between sex-specific masticatory features and dentoskeletal morphological features would not indicate whether morphological features caused the chewing differences or vice versa. The adult dentoskeletal anatomy plays an important role in shaping the chewing cycle (see Bates et al., 1975). However, there is evidence that neuromuscular activity, i.e., oral function, plays an important role in shaping the dentoskeletal morphology (Byrd et al., 1990). Hence, dentoskeletal and muscular differences that exist between male and female subjects (Ingerslev and Solow, 1975; Tradowsky, 1990; Terk and Fenart, 1992) may either have caused or been caused by functional masticatory differences.

It has recently been proposed that the developing organism elaborates specific motor patterns based on a dynamic interaction between inherited phenotypic predispositions

Table 5. Jackknifed classification matrix

\begin{tabular}{lccc}
\hline Group & $\%$ Correct & Female Classifications & Male Classifications \\
\hline Female & 94.1 & 16 & 1 \\
Male & 92.9 & 1 & 13 \\
Total & 93.5 & & \\
\hline
\end{tabular}

within the organism's sensorimotor systems and the environment with which the organism interacts (Thelen and Smith, 1994). If the oral apparatus is similar to other well-studied systems involved in motor production, then it is possible that masticatory sex specificity emerges as a result of dynamic interactions between genetically inherited potentialities and social and cultural pressures interacting with the oral apparatus through oral function. In other words, based on the recent proposal for motor development (Thelen and Smith, 1994), we hypothesize that the dynamic interaction of genetic, epigenetic, social, and cultural pressures results in sex-specific developmental bifurcations in the dentoskeletal and neuromuscular systems generating mastication.

The point is that these new ideas of developmental motor acquisition can be tested rigorously on oral function with a combination of the methods introduced in the current paper and the established methods of cephalometrics or dentoskeletal morphometrics. Hence, complex multidimensional links between morphology and function can be evaluated with relatively sophisticated techniques. Future studies will be able to study the co-development of form and function; such studies will lend considerable insight into when and how sex-specific differences emerge within the dentoskeletal and neuromotor systems.

It is known that aspects of jaw function and parafunction (Christensen, 1971; Davidson and Mohl, 1987; Klineberg 1988) are associated with temporomandibular disorders (TMD). Furthermore, current theories of TMD etiology agree that muscle hyperactivity is probably the final common pathway leading to TMD pain symptoms (Rugh, 1981; Zarb and Mohl, 1988). Since TMD are more common in females (Locker and Slade, 1988; Huber and Hall, 1990; Bakke and Møller, 1992; Krogstad et al., 1992; De Kanter et al., 1993; however, cf. Duckro et al., 1990; Glass et al., 1993), this means that sex-specific risk factors associated with oral function predispose women to developing TMD. This being the case, we hypothesize that some of these functional risk factors may have been quantified in the variables defined in Table 2. Future studies will need to determine whether chewing pattern aspects that are more common among women represent risk factors associated with TMD.

On the other hand, women probably adapt to orofacial pain differently than do men, and this could be one reason why TMD appears to be more common in women. However, mild TMD symptoms affect up to $80 \%$ of Western populations (Rugh and Solberg, 1985; Rugh, 1991). Moreover, adaptive responses to orofacial pain involve changes in oral motor output (Stohler et al., 1988). Consequently, for a large portion of the population, oral function has probably been influenced in sex-specific ways by pain symptoms. This again suggests that future 
Table 6. Pearson's coefficients of correlation between specific variables and subject height

\begin{tabular}{lcc}
\hline Variable Name $^{\mathrm{a}}$ & Female Group $(\mathrm{n}=17)$ & Male Group (n=14) \\
\hline Latency to max. acceleration during closing + cycle duration (row 17) & $\mathrm{r}=0.262$ & $\mathrm{r}=-0.400$ \\
& $\mathrm{p}=0.310$ & $\mathrm{p}=0.156$ \\
Angle $\theta_{\mathrm{a}-\mathrm{p}} @$ mc2 (row 28) & $\mathrm{r}=0.54$ & $\mathrm{r}=-0.004$ \\
& $\mathrm{p}=0.837$ & $\mathrm{p}=0.989$ \\
\hline
\end{tabular}

a Rows correspond to variable locations in Table 2.

investigations will need to look at the relationship between sexspecific oral function and orofacial pain.

In summary, the current study found that mastication of female subjects could be distinguished from that of male subjects. The distinction was less pronounced for univariate measures than for multidimensional discriminant functions. These results differ from those of previous work where significant sex-specific differences were found among univariate measures. It is likely that for gum chewing, sex specificity is minimal, appearing primarily as variation within an ensemble of variables, whereas for other food substances, sex specificity is more pronounced at the univariate level. Further work is needed to gain precise and detailed insight into sex-based differences in oral functional movements. Work is also required to evaluate how generalizable the current study's findings are, whether the results represent genetically inherited or socially acquired differences, and how these differences affect and are affected by the dentoskeletal system, disease processes, lifestyles, and social/cultural pressures.

\section{Acknowledgments}

We wish to thank Michael Corrigan, Darlene Tansil, and Reena Shah for help with data entry, and Darlene Tansil for help with artwork. We also wish to thank Dr. Charles J. Kowalski, Statistical Research Unit, Department of Biologic and Materials Sciences, School of Dentistry, University of Michigan, for his careful reading of the manuscript and helpful suggestions. This work was supported by USPHS Research Grant R29 DE-10625 from the National Institute of Dental Research, National Institutes of Health, Bethesda, MD 20892.

A preliminary report was presented at the 1995 American Association for Dental Research Meeting, San Antonio, TX.

\section{References}

Aboitiz F, Scheibel AB, Zaidel E (1992). Morphometry of the Sylvian fissure and the corpus callosum, with emphasis on sex differences. Brain 115(Pt 5):1521-1541.

Alexander GM, Hines M (1994). Gender labels and play styles: their relative contribution to children's selection of playmates. Child Dev 65:869-879.

Allen LS, Gorski RA (1990). Sex difference in the bed nucleus of the stria terminalis of the human brain. J Comp Neurol 302:697-706.

Allen LS, Gorski RA (1992). Sexual orientation and the size of the anterior commissure in the human brain. Proc Natl Acad Sci 89:7199-7202.

Allen LS, Richey MF, Chai YM, Gorski RA (1991). Sex differences in the corpus callosum of the living human being. J Neurosci 11:933-942.

Ames FR (1991). Sex and the brain. S Afr Med J 80:150-152.

Bakke M (1993). Mandibular elevator muscles: physiology, action, and effect of dental occlusion. Scand J Dent Res 101:314-331.

Bakke M, Møller E (1992). Craniomandibular disorders and masticatory muscle function. Scand J Dent Res 100:32-38.

Bakke M, Holm B, Jensen BL, Michler L, Möller E (1990). Unilateral, isometric bite force in 8-68-year-old women and men related to occlusal factors. Scand J Dent Res 98:149-158.

Bates JF, Stafford GD, Harrison A (1975). Masticatory functiona review of the literature. 1 . The form of the masticatory cycle. J Oral Rehabil 2:281-301.

Byrd KE, Stein ST, Sokoloff AJ, Shankar K (1990). Craniofacial alterations following electrolytic lesions of the trigeminal motor nucleus in actively growing rats. Am J Anat 189:93-110.

Christensen LV (1971). Facial pain and internal pressure of masseter muscle in experimental bruxism in man. Arch Oral Biol 16:1021-1031.

Corruccini RS, Henderson AM, Kaul SS (1985). Bite-force variation related to occlusal variation in rural and urban Punjabis (North India). Arch Oral Biol 30:65-69.

Davidson RM, Mohl ND (1987). Sensory and motor integration during mandibular function. Dent Clin N Am 31:615-626.

De Kanter RJ, Truin GJ, Burgersdijk RC, Van ' $t$ Hof MA, Battistuzzi PG, Kalsbeek H, et al. (1993). Prevalence in the Dutch adult population and a meta-analysis of signs and symptoms of temporomandibular disorder. / Dent Res 72:1509-1518.

Dixon WJ, editor (1992). Stepwise discriminant analysis. In: BMDP statistical software manual. Berkeley: University of California Press, pp. 363-385.

Duckro PN, Tait RC, Margolis RB, Deshields TL (1990). Prevalence of temporomandibular symptoms in a large United States metropolitan area. Cranio 8:131-138.

Dworkin SF, LeResche L (1992). Research diagnostic criteria for temporomandibular disorders: review, criteria, examinations and specifications, critique. J Craniomandib Disord Facial Oral Pain 6:301-355.

Fetz EE (1992). Are movement parameters recognizably coded in the activity of single neurons? Behav Brain Sci 15:679-690.

Gerstner GE (1992). Quantifiable temporal and spatial behavior patterns in six mammalian species: insights into ultimate and proximate causality (dissertation). Los Angeles, CA: University of California.

Gerstner GE, Goldberg LJ (1989). An analysis of mandibular movement trajectories and masticatory muscle EMG activity during drinking in the guinea pig. Brain Res 479:6-15.

Gerstner GE, Goldberg LJ (1991). Genioglossus EMG activity 
during rhythmic jaw movements in the anesthetized guinea pig. Brain Res 562:79-84.

Gerstner GE, Goldberg LJ (1994). Species-specific morphology of masticatory jaw movements. Behaviour 128:229-253.

Gerstner GE, Goldberg LJ, DeBruyne K (1989). Angiotensin IIinduced rhythmic jaw movements in the ketamineanesthetized guinea pig. Brain Res 478:223-240.

Glass EG, McGlynn FD, Glaros AG, Melton K, Romans K (1993). Prevalence of temporomandibular disorder symptoms in a major metropolitan area. Cranio 11:217-220.

Goldberg S, Lewis M (1969). Play behavior in the year-old infant: early sex differences. Child Dev 40:21-31.

Gorski RA (1978). Sexual differentiation of the brain. Hosp Pract 13:55-62.

Gorski RA (1984). Critical role for the medial preoptic area in the sexual differentiation of the brain. Prog Brain Res 61:129-146.

Gorski RA, Gordon JH, Shryne JE, Southam AM (1978). Evidence for a morphological sex difference within the medial preoptic area of the rat brain. Brain Res 148:333-346.

Goulet JP, Clark GT (1990). Clinical TMJ examination methods. J CA Dent Assoc 18:25-33.

Hines M, Kaufman FR (1994). Androgen and the development of human sex-typical behavior: rough-and-tumble play and sex of preferred playmates in children with congenital adrenal hyperplasia (CAH). Child Dev 65:1042-1053.

Hines M, Alsum P, Roy M, Gorski RA, Goy RW (1987). Estrogenic contributions to sexual differentiation in the female guinea pig: influences of diethylstilbestrol and tamoxifen on neural, behavioral, and ovarian development. Horm Behav 21:402-417.

Hines M, Allen LS, Gorski RA (1992). Sex differences in subregions of the medial nucleus of the amygdala and the bed nucleus of the stria terminalis of the rat. Brain Res 579:321-326.

Howell PG (1987). Sexual dimorphism in mastication and speech? Or do men and women eat and talk differently? Aust Prostho J 1:9-17.

Huber MA, Hall EH (1990). A comparison of the signs of temporomandibular joint dysfunction and occlusal discrepancies in a symptom-free population of men and women. Oral Surg Oral Med Oral Pathol 70:180-183.

Hutt C (1978). Biological bases of psychological sex differences. Am J Dis Child 132:170-177.

Ingerslev $\mathrm{CH}$, Solow B (1975). Sex differences in craniofacial morphology. Acta Odontol Scand 33:85-94.

Klineberg I (1988). Occlusion as the cause of undiagnosed pain. Int Dent J 38:19-27.

Korner AF (1973). Sex differences in newborns with special reference to differences in the organization of oral behavior. J Child Psychol Psychiatry 14:19-29.

Krogstad BS, Dahl BL, Eckersberg T, Øgaard B (1992). Sex differences in signs and symptoms from masticatory and other muscles in 19-year-old individuals. J Oral Rehabil 19:435-440.

Lobel TE (1994). Sex typing and the social perception of gender stereotypic and nonstereotypic behavior: the uniqueness of feminine males. J Pers Soc Psychol 66:379-385.

Locker D, Slade G (1988). Prevalence of symptoms associated with temporomandibular disorders in a Canadian population. Community Dent Oral Epidemiol 16:310-313.
Long RE Jr, Jain RB, Krogman WM (1982). Possible sexdiscriminant variables in craniofacial growth in clefting. Am J Orthod 82:392-402.

Neill DJ, Howell PG (1986). Computerized kinesiography in the study of mastication in dentate subjects. J Prosthet Dent 55:629-638.

Phillips G, Over R (1992). Adult sexual orientation in relation to memories of childhood gender conforming and gender nonconforming behaviors. Arch Sex Behav 21:543-558.

Raum WJ, McGivern RF, Peterson MA, Shryne JH, Gorski RA (1990). Prenatal inhibition of hypothalamic sex steroid uptake by cocaine: effects on neurobehavioral sexual differentiation in male rats. Brain Res Dev Brain Res 53:230-236.

Rugh JD (1981). Psychological stress in orofacial neuromuscular problems. Int Dent J 31:202-205.

Rugh JD (1991). Behavioral therapy for temporomandibular disorders. Curr Opin Dent 1:497-502.

Rugh JD, Solberg WK (1985). Oral health status in the United States: Temporomandibular disorders. J Dent Educ 49:398-406.

Rugh JD, Harlan J (1988). Nocturnal bruxism and temporomandibular disorders. Adv Neurol 49:329-341.

Schiffman EL, Fricton JR, Haley D (1992). The relationship of occlusion, parafunctional habits and recent life events to mandibular dysfunction in a non-patient population. J Oral Rehabil 19:201-223.

Seligman DA, Pullinger AG (1991). The role of functional occlusal relationships in temporomandibular disorders: a review. J Craniomandib Disord 5:265-279.

Sheikholeslam A, Möller E, Lous I (1980). Pain, tenderness and strength of human mandibular elevators. Scand I Dent Res 88:60-66.

Stohler CS, Ashton-Miller JA, Carlson DS (1988). The effects of pain from the mandibular joint and muscles on masticatory motor behaviour in man. Arch Oral Biol 33:175-182.

Stoner $S$ (1978). Sex differences in responses of children to the hand test. Percept Mot Skills 46(Pt 1):759-762.

Thelen E, Smith LB (1994). A dynamic systems approach to the development of cognition and action. Cambridge: The MIT Press.

Tradowsy (sic) M (1990). Sex difference in intercondylar distance. J Prosthet Dent 63:301-302.

Terk B, Fenart R (1992). Differences sexuelles des segments et angles du profil facial, etudiées par l'analyse discriminante [Sex differences of segments and angles of the facial profile, as studied by discriminant analysis]. Orthod Fr 2:613-617.

Trenouth MJ (1979). The relationship between bruxism and temporomandibular joint dysfunction as shown by computer analysis of nocturnal tooth contact patterns. $J$ Oral Rehabil 6:81-87.

Weisfeld GE (1979). An ethological view of human adolescence. JNerv Ment Dis 167:38-55.

Zarb GA, Mohl ND (1988). Occlusion and temporomandibular disorders: a prologue. In: A textbook of occlusion. Mohl ND, Zarb GA, Carlsson GE, Rugh JD, editors. Chicago: Quintessence, pp. 377-383.

Zucker KJ, Doering RW, Bradley SJ, Finegan JK (1982). Sextyped play in gender-disturbed children: a comparison to sibling and psychiatric controls. Arch Sex Behav 11:309-321.

Zuger B (1984). Early effeminate behavior in boys: outcome and significance for homosexuality. J Nerv Ment Dis 172:90-97. 